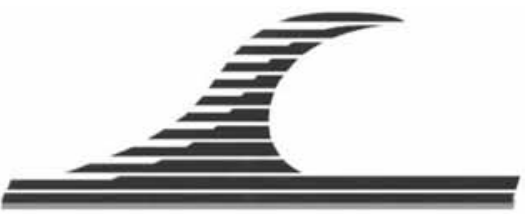

Mots-clés : Sédiment marin, Propriétés physiques et chimiques, Sédimentation, Consolidation, Tassement, Perméabilité,

\title{
Valorisation en unité pilote de sédiments méditerranéens : étude des caractéristiques géotechniques et de la perméabilité
}

\author{
Daniel LEVACHER ${ }^{1}$, Martin SANCHEZ ${ }^{2}$, Zhibo DUAN ${ }^{3}$, Yingjie LIANG $^{1}$
}

1. Université de Caen, Faculté des Sciences, UMR 6143 CNRS - M2C, Esplanade de la Paix, 14032 Caen, France.

daniel.levacher@unicaen.fr; yingjie.liang@unicaen.fr

2. Université de Nantes, UMR 6112 CNRS - Planétologie et Géodynamique, 2 rue de la Houssinière, 44322 Nantes, France.

martin.sanchez@univ-nantes.fr

3. Ecole Centrale de Lille, UMR 8107, Laboratoire de Mécanique de Lille,

Bd Paul Langevin, 59655 Villeneuve d'Ascq cedex, France.

zhibo.duan@ec-lille.fr

\section{Résumé :}

Les sédiments marins trouvent leur réemploi avec ou sans traitement dans la construction d'ouvrages portuaires et routiers. Ces applications imposent toute une méthodologie pour leur caractérisation hydraulique et mécanique. Cette méthodologie est proposée pour le projet SEDIMARD 83 et appliquée à des sédiments marins prélevés sur 6 sites portuaires de la côte Méditerranéenne. Après une caractérisation géotechnique élémentaire (teneurs en eau initiale, granulométrie, limites d'Atterberg, teneurs en carbonates et matières organiques) les relations perméabilité - concentration (ou indice des vides) sont établies à partir d'essais de décantation en colonne et d'essais de consolidation œdomètrique. Les essais de décantation en colonne correspondent au tassement sous poids propre du sédiment. Les essais de consolidation œdométrique correspondent au tassement de ces mêmes sédiments sous surcharges. Tout d'abord, l'étude des propriétés physiques et chimiques des sédiments montre qu'elles varient peu pour une même région d'origine (Méditerranée). Cependant, un traitement de tri et de séparation (sédiment brut/sédiment dessablé/déshydraté) les modifie.

L'analyse des résultats des essais de décantation et de consolidation a conduit à l'obtention des paramètres classiques de tassement mais aussi à la définition de la relation entre la perméabilité et l'indice des vides. Une continuité a été bien observée entre les résultats issus de ces deux essais.

Article lié au projet SEDIMARD 83 -Partie II-

Soumis le 23 février 2009, accepté le 11 janvier 2011, en ligne le 4 avril 2011.

La seule version examinée est celle écrite en français. La ou les autres versions n'étant pas examinées par le comité de rédaction de la revue, sont donc publiées sous l'entière responsabilité du ou des auteurs.

A TRANSLATED VERSION IN ENGLISH IS AVAILABLE ONLINE

Pour citer cet article :

LEVACHER D., SANCHEZ M., DUAN Z., LIANG Y. (2011). Valorisation en unité pilote de sédiments méditerranéens : étude des caractéristiques géotechniques et de la perméabilité. Revue Paralia, Vol. 4, pp 4.1-4.20. 


\section{2 : Revue Paralia - Vol. 4 (2011)}

\section{Introduction}

Valoriser les sédiments marins constitue un enjeu important dans leur gestion. Les récents travaux de recherche à ce sujet sont très orientés "matériaux routiers et matériaux de construction". Les objectifs visés dans ces travaux sont ambitieux connaissant toutes les difficultés à stabiliser et/ou solidifier les sédiments marins ou fines de barrages et les échecs quant à l'application sur site. Face à ces difficultés les travaux de COLIN (2003) et LEMEE (2006) réalisés sur des sédiments fluviaux et marins prennent bien en compte l'aspect économique du problème. SEMCHA (2006) a même mis au point un matériau de construction (tuile) à partir de fines de barrage. Finalement la valorisation des matériaux fins dragués peut être hiérarchisée en fonction des différents objectifs à atteindre (LEVACHER et al., 2007a ; tableau 1).

Tableau 1. Hiérarchisation des objectifs d'ouvrabilité et de réutilisation des sédiments et fines de barrage et domaines d'application concernés (LEVACHER et al., 2007a).

\begin{tabular}{ll}
\hline Objectif & Application et domaine concerné \\
\hline $\begin{array}{l}\text { 1-Pelletabilité du produit en cours de } \\
\text { stabilisation/solidification. }\end{array}$ & $\begin{array}{l}\text { Transport et mise en décharge (matériau fin } \\
\text { contaminé), mise en dépôt pour stockage. }\end{array}$ \\
\hline $\begin{array}{l}\text { 2-Mise en dépôt : remplissage de carrières, } \\
\text { de cavités souterraines. }\end{array}$ & \begin{tabular}{l} 
Matériau de remplissage, de comblement. \\
\hline 3-Réalisation de remblai sans surcharge.
\end{tabular} \\
\hline 4-Remblai avec surcharge. & $\begin{array}{l}\text { Consolidation sous poids propre, zones } \\
\text { aménagées }\end{array}$ \\
\hline 5-Valorisation en technique routière. & $\begin{array}{l}\text { Matériau de terre-pleins et de plateformes } \\
\text { industriels. }\end{array}$ \\
\hline 6-Valorisation en matériaux élaborés. & Matériau de sous-couches routières. \\
\hline
\end{tabular}

Toute valorisation relative à la réutilisation des sédiments marins en remblai impose une caractérisation de paramètres géotechniques et "chimiques" de base du matériau ainsi que de ses propriétés hydrauliques, de consolidation et de résistance (LEVACHER et al., 2005).

La caractérisation géotechnique de base des sédiments naturels faisant l'objet de valorisation comprend :

- la teneur en eau,

- la répartition dimensionnelle des grains et particules par granulométrie et/ou sédimentométrie,

- la teneur en argiles $(<2 \mu \mathrm{m})$,

- les limites d'Atterberg et indice de plasticité et/ou mesure de valeur au bleu. 
Les classes granulaires des sédiments naturels faisant l'objet de valorisation quelle qu'en soit la finalité vont de 0 à $5 \mathrm{~mm}$ comprenant les fines et les sables. Au-delà de cette taille maximale, les matériaux grossiers contenus dans les sédiments peuvent être réutilisés comme granulats. La classe granulaire des sédiments étudiés pour une réutilisation en remblai est très souvent de $0 / 2 \mathrm{~mm}$.

Les propriétés hydrauliques, de consolidation et de résistance sont établies à partir des essais de décantation à la colonne, de consolidation, de consolidation adométrique. Elles comprennent respectivement :

- les relations perméabilité-concentration $(k-C)$ et loi de décantation,

- les relations perméabilité-concentration $(k-C)$ pour les matériaux consolidés, les coefficients de consolidation et fluage, les indices de compression et de gonflement,

- les relations teneur en eau-cohésion $(\mathrm{W}-\mathrm{Cu})$, ou concentration $(\mathrm{C}-\mathrm{Cu})$.

Les caractérisations chimiques comprennent :

- la teneur en en carbonates,

- la teneur en matières organiques,

- la teneur en contaminants.

La nature et la teneur de constituants dans les sédiments sont des éléments clés dans la recherche de techniques de stabilisation. En effet la présence de carbonates ou de silice peut être intéressante pour l'utilisation de traitements chimiques à base de polymères ou réactifs (LEMEE, 2006 ; LIANG, 2008). La présence de matières organiques amènera des effets perturbateurs dans les stabilisations aux liants hydrauliques. Et bien sûr, les teneurs en contaminants doivent être connues pour envisager toute opération de traitement sur des sédiments pollués.

Finalement, ces caractérisations apportent tous les éléments nécessaires à une valorisation des sédiments en remblai sans traitement (remblai confiné ou non) et permet d'envisager un traitement de stabilisation et/ou de solidification avec additifs. Pour les autres filières avec traitement, de stabilisation et/ou solidification, un second volet d'études s'impose qui comprend différentes phases de validation d'indicateurs d'ouvrabilité (compactage et mécanique), comportementaux et économiques (SANNIER et al., 2008).

Les sédiments étudiés proviennent de ports de la côte méditerranéenne. Ils ont fait l'objet d'une caractérisation géotechnique minimale destinée à l'orientation vers des filières de valorisation en génie civil: ouvrages en terre (remblais) et matériaux (construction). Cette caractérisation de base est physico-mécanique, c'est-à-dire que les propriétés chimiques liées principalement au degré de contamination ne font pas partie de l'étude et que le comportement environnemental des sédiments étudiés n'est pas abordé (LEVACHER et al., 2007a ; LEVACHER et al., 2007b ; LEVACHER et al., 2008). Cette caractérisation porte sur les principales propriétés géotechniques (teneurs en eau $W$, limites d'Atterberg, granulométrie) et chimiques (teneurs en carbonates et matières organiques $M O$ ). 
Un des objectifs de l'étude est de caractériser et d'identifier des groupes de sédiments d'origines diverses de manière à optimiser les processus de traitement par groupe de sédiment et non pas individuellement par sédiment.

\section{Matériaux et méthodes}

Les matériaux étudiés sont des sédiments de dragage provenant de l'Arsenal de Toulon, des ports de Bandol, de Cannes, de Sanary-Lavandou, de Saint-Mandrier et du Vieux Port de Marseille. Après dragage, ils ont été amenés sur un site "pilote" pour y être traités et éventuellement valorisés. Ce site "pilote" a fait l'objet à part entière du projet "SEDIMARD 83" (GROSDEMANGE et al., 2008).

Les matériaux analysés proviennent du site "pilote", ils sont issus de 2 types d'échantillonnage :

- les sédiments naturels ou bruts prélevés à partir du bassin de stockage de ce site de traitement, ayant subi un dégrillage (élimination des macro-déchets), un criblage à sec $(0 / 50 \mathrm{~mm})$;

- les sédiments prélevés après les opérations de dessablage (criblage humide à $3 \mathrm{~mm}$, puis séparation granulométrique par hydrocyclonage) et de déshydratation par filtrepresse.

Aux 6 sites de prélèvement correspondent donc 12 échantillons (bruts et dessablés). Les matériaux dans leur ensemble, ont été prélevés et acheminés en laboratoire à deux différentes dates : les 19 mars 2007 et 06 mars 2008. Le transport et la conservation des matériaux ont été effectués en bidons étanches en plastique opaque, d'une contenance de 201 . Après acheminement en laboratoire les matériaux ont été analysés dès leur arrivée : propriétés physiques et chimiques. Puis les essais de décantation et de consolidation ont suivi.

Chaque échantillon est référencé et repéré suivant le lieu de dragage, son état brut ou dessablé-déshydraté, l'année d'analyse, de la manière suivante (tableau 2) :

- lieu de prélèvement "ABC/", trois lettres dénommant respectivement le lieu de prélèvement c'est-à-dire le port d'origine,

- type d'échantillons "brut" ou "dessablé et déshydraté", respectivement dénommé BRUT ou DD,

- année de prélèvement 2007 ou 2008, mentionnée par la suite dans les sections relatives à l'évolution temporelle des propriétés des sédiments.

L'absence de certains sédiments en 2008 résulte du fait qu'au niveau du site "pilote", ils étaient déjà prétraités (absence de sédiments bruts) ou entièrement valorisés (cas du sédiment de Saint-Mandrier). 
Valorisation en unité pilote de sédiments méditerranéens : étude des caractéristiques géotechniques et de la perméabilité : 4.5

Tableau 2. Récapitulatif des origines, références des sédiments et dates de réception.

\begin{tabular}{llll}
\hline Port d'origine & Références des sédiments Date de réception & Date de réception \\
\hline Arsenal de Toulon & ARS/BRUT & Mars 2007 & Mars 2008 \\
Arsenal de Toulon & ARS/DD & Mars 2007 & Mars 2008 \\
Bandol & BAN/BRUT & Mars 2007 & - \\
Bandol & BAN/DD & Mars 2007 & Mars 2008 \\
Cannes & CAN/BRUT & Mars 2007 & - \\
Cannes & CAN/DD & Mars 2007 & Mars 2008 \\
Sanary-Lavandou & SAN/BRUT & Mars 2007 & - \\
Sanary-Lavandou & SAN/DD & Mars 2007 & Mars 2008 \\
Saint-Mandrier & $S T M / B R U T$ & Mars 2007 & - \\
Saint-Mandrier & $S T M / D D$ & Mars 2007 & - \\
Vieux Port de Marseille & VPM/BRUT & Mars 2007 & - \\
Vieux Port de Marseille & VPM/DD & Mars 2007 & Mars 2008 \\
\hline
\end{tabular}

Les méthodes de détermination des caractéristiques physiques sont classiques et respectent les normes en vigueur.

La teneur en eau est obtenue par séchage en étuve à $105{ }^{\circ} \mathrm{C}$ selon la norme (NF P 94-050). Les teneurs en eau ont été déterminées dès la réception des bidons de sédiments à la date de leur réception en laboratoire pour tous les sédiments. Lors de manipulations, il convient d'homogénéiser le sédiment avant toute mesure de teneur en eau.

Les limites d'Atterberg sont des teneurs en eau qui délimitent des comportements critiques des matériaux: limites de liquidité $W_{L}$ et de plasticité $W_{P}$ (méthode de la coupelle et du rouleau), selon la norme NF P 94-051. La finesse des matériaux dont la totalité des passants à $2 \mathrm{~mm}$ est supérieure à $95 \%$ a permis de réaliser des granulométries laser sur l'ensemble des sédiments. L'appareil utilisé, un Coulter LS230, permet d'explorer une large gamme de particules allant de 0.04 à $2000 \mu \mathrm{m}$. Dans le cas de la présence de particules grossières, un tamisage à sec est opéré jusqu'à $5 \mathrm{~mm}$. Les teneurs en matières organiques ont été déterminées à partir de la mesure du pourcentage pondéral de matière organique M.O. après calcination des matériaux à $550{ }^{\circ} \mathrm{C}$ sur une durée de 4h selon la norme XP P 94-047. Cette méthode par perte au feu ou perte par calcination est un des moyens d'apprécier l'état de décomposition des matières organiques. L'utilisation d'un calcimètre Bernard permet l'obtention des teneurs en carbonates conformément à la norme NF P 94-048.

Les propriétés hydrauliques font appel aux techniques usuelles utilisées pour les études de sédimentation et de consolidation de sols fins saturés. Ces propriétés fournissent les paramètres nécessaires aux calculs de tassement pour la mise en remblai des sédiments traités ou non (relation entre la perméabilité $k$ et la concentration en matières solides $C$ ). 
Les essais de décantation sont effectués pour des concentrations initiales différentes et représentatives des sédiments prélevés selon les modes opératoires de dragage.

L'essai à la colonne de décantation consiste en la mise en œuvre d'un mélange de matériau fin homogène de concentration initiale $C i$ dans un tube transparent de diamètre extérieur de $54 \mathrm{~mm}$, épais de $2 \mathrm{~mm}$ et de hauteur totale de $2 \mathrm{~m}$ pour les faibles concentrations $(50 \mathrm{~g} / \mathrm{l})$. Pour les concentrations de $250 \mathrm{~g} / \mathrm{l}$, les tubes transparents ont les caractéristiques suivantes : diamètre $94 \mathrm{~mm}$, épaisseur de $3 \mathrm{~mm}$ et hauteur de $2 \mathrm{~m}$. Pendant le processus de décantation, l'évolution de la hauteur d'échantillon est suivie en fonction du temps par des mesures très rapprochées pour les six premières heures, à savoir : $30 \mathrm{~s}, 1 \mathrm{mn}, 2 \mathrm{mn}, 4 \mathrm{mn} 6 \mathrm{mn} \ldots 30 \mathrm{mn}, 35 \mathrm{mn}, 40 \mathrm{mn}, \ldots 1 \mathrm{~h}, 1 \mathrm{~h} 10,1 \mathrm{~h} 20$, ... et $2 \mathrm{~h}, 2 \mathrm{~h} 20,2 \mathrm{~h} 40,3 \mathrm{~h}, 3 \mathrm{~h} 30,4 \mathrm{~h}, 5 \mathrm{~h}, 6 \mathrm{~h}, 24 \mathrm{~h}$, puis tous les $24 \mathrm{~h}$. La période d'observation a été de 10 jours. En l'absence de contraintes effectives, la vitesse de sédimentation ou de décantation $V s$ d'une vase ou d'un sédiment fin reposant sur un fond imperméable (négative pour désigner une chute) sous l'action de son propre poids peut être calculée à l'aide de la relation de BEEN (1980) suivante :

$V_{s}=-\frac{k}{\rho_{S}}\left[\frac{\rho_{S}}{\rho_{o}}-1\right] C$

Dans laquelle $\rho_{s}$ est la masse volumique de la phase solide (de l'ordre de $2550 \mathrm{~kg} \mathrm{~m}^{-3}$ pour une vase ou sédiment fin organique), $\rho_{o}$ est la masse volumique de l'eau (prise égale à $1000 \mathrm{~kg} \mathrm{~m}^{-3}$ ), $C$ est la concentration en matières sèches (rapport de la masse solide sur le volume total), $k$ est le coefficient de perméabilité. Sachant que le coefficient de perméabilité d'une vase ou d'un sédiment fin donné dépend de sa concentration, la théorie de sédimentation de KYNCH (1952) est compatible avec la formulation de BEEN (1980). Des travaux réalisés par SANCHEZ (2000) montrent que dans le cas de vases molles, la relation entre $k$ et $C$ obéit fréquemment à la loi empirique suivante :

$$
k=A_{1} \exp \left(-A_{2} \frac{C}{\rho_{s}}\right)
$$

où $A_{1}\left(\mathrm{~m} \mathrm{~s}^{-1}\right)$ et $A_{2}$ sont des paramètres propres à chaque vase ou sédiment fin. Les paramètres de la loi précédente peuvent être évalués directement à partir de courbes expérimentales de tassement par une méthode graphique développée par KYNCH (1952) et modifiée par SANCHEZ \& GROVEL (1993).

La consolidation sous charge verticale a été étudiée à l'œdométre, essai classique de la mécanique des sols. Sachant que cet essai s'applique aux sols fins à teneur en eau bien inférieure à celle des sédiments marins, l'échantillon testé a fait l'objet d'un premier chargement avec une contrainte de consolidation faible de $12.5 \mathrm{kPa}$, puis $25,50,100$, 200 et $400 \mathrm{kPa}$ pour tous les essais. 
Valorisation en unité pilote de sédiments méditerranéens : étude des caractéristiques géotechniques et de la perméabilité : 4.7

\section{Résultats}

\subsection{Caractéristiques géotechniques des sédiments}

\subsubsection{Granulométries des sédiments}

Les courbes granulométriques de tous les échantillons sont bien réparties entre $1 \mu \mathrm{m}$ et $5 \mathrm{~mm}$. Les granulométries réalisées sur les sédiments bruts et dessablés/déshydratés notés DD montrent qu'il n'y a pas de variation de forme de la courbe granulométrique et les six sédiments sont relativement proches (figure 1). Le fuseau le plus étroit concerne les sédiments pré traités à savoir les dessablés et déshydratés.

La fraction argileuse $(<2 \mu \mathrm{m})$ représente 5 (SAN, BAN, CAN) à 15\% (ARS) des sédiments. Elle est stabilisée autour de 10\% pour les sédiments DD. La fraction silteuse (2-20 $\mu \mathrm{m})$ représente 20 à $45 \%$ des matériaux bruts. Elle est stabilisée entre 30 et $40 \%$ pour les matériaux DD. Le sédiment le plus sableux est celui référencé en SAN. Les sédiments les plus argileux sont ceux de l'Arsenal de Toulon (ARS) et du Vieux Port de Marseille (VPM), voir figure 1.

Si l'on considère les analyses granulométriques des sédiments prélevés sur les deux périodes 2007 et 2008, les distributions recueillies pour chaque sédiment montrent qu'il n'y a pas eu de modifications granulaires quel que soit le sédiment étudié. Ceci est illustré à la figure 2 pour un sédiment brut et dessablé/déshydraté de l'Arsenal de Toulon.

\subsubsection{Teneurs en eau initiales des sédiments}

Le cycle de tri granulaire et de séparation solide-liquide appliqué est présenté sur le schéma de la figure 3. Il permet de faire passer le sédiment de l'état brut (bassin de stockage, figure 3) à l'état de dessablé-déshydraté (DD) à la fin du cycle. Des mesures de teneurs en eau $W$, ont pu être effectuées durant ce cycle sur les sédiments bruts et dessablés-déshydratés à des dates différentes correspondant aux prélèvements de 2007 et de 2008 (voir tableau 3). Des mesures de teneurs ont été relevées par SANNIER (2008) au moment des dragages en 2007 sur les sédiments bruts. Ainsi quatre étapes de mesure de $W$ sont rapportées dans le tableau 3 :

1) au moment des dragages, mesures sur site en 2007 , une série de sédiments bruts,

2) prélèvement au niveau du pilote en 2007 , mesures en 2007 , avec deux séries pour comparaison brut-dessablé,

3) prélèvement au niveau du pilote en 2007, mesures en 2008, avec deux séries pour comparaison brut-dessablé,

4) prélèvement au niveau du pilote en 2008, mesures en 2008, une série de sédiments bruts (sans STM) et deux valeurs pour le sédiment ARS pour comparaison brutdessablé. 

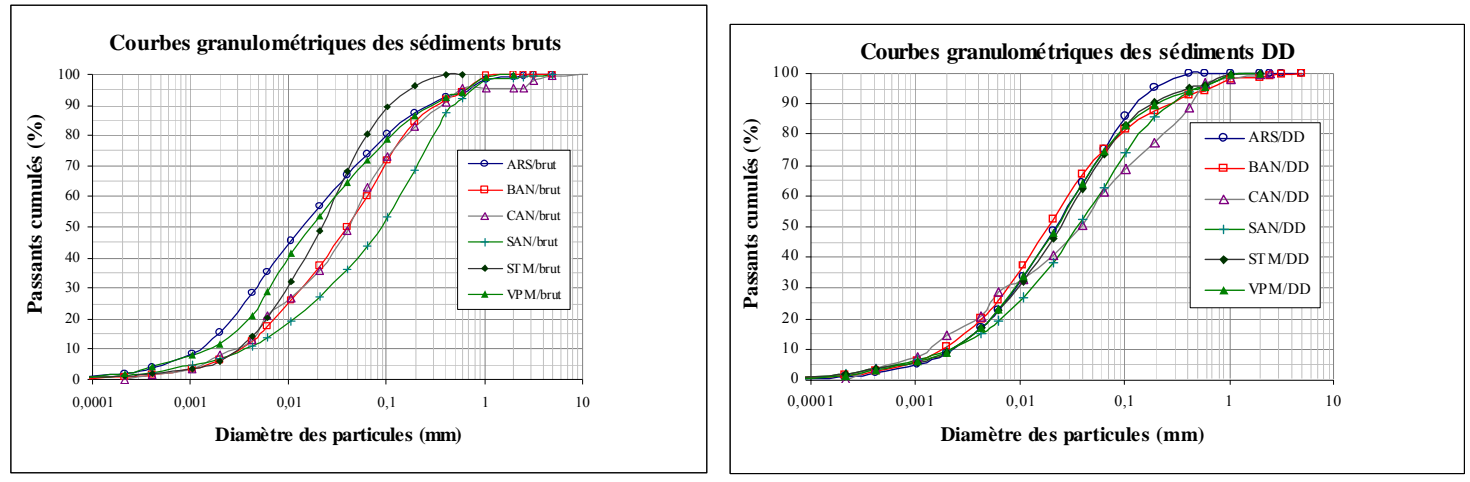

Figure 1. Granulométries des sédiments bruts et dessablés/déshydratés.
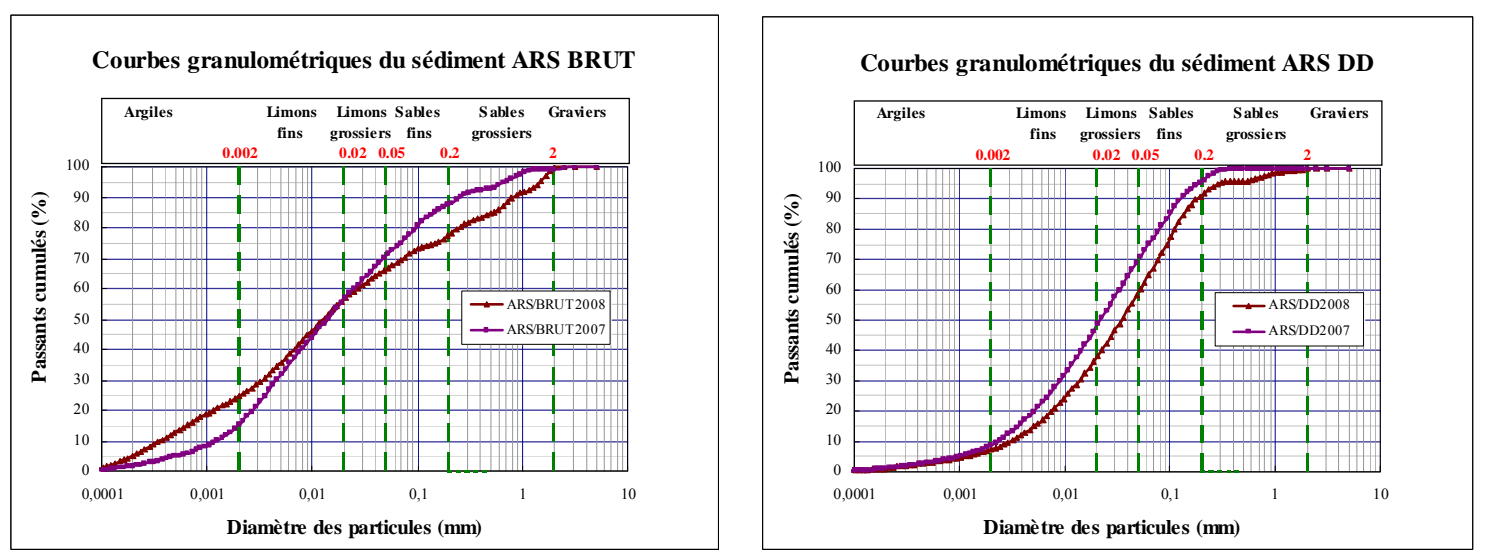

Figure 2. Evolution des distributions des sédiments prélevés en 2007 et en 2008.

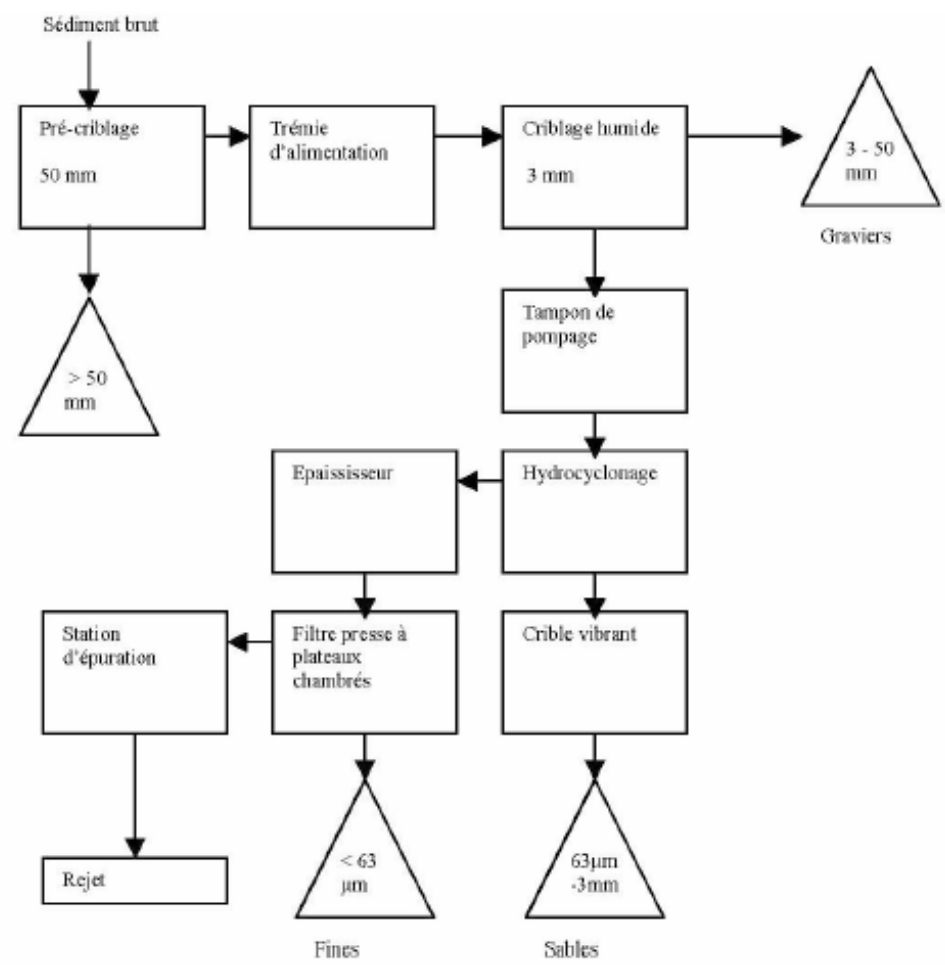

Figure 3. Les cycles de prétraitements de l'unité pilote. 
Valorisation en unité pilote de sédiments méditerranéens : étude des caractéristiques géotechniques et de la perméabilité : 4.9

Les teneurs en eau ont été déterminées dès la réception des bidons de sédiments à la date du 19 mars 2007 pour les deux séries de sédiments. Elles ont été de nouveau mesurées le 6 mars 2008 avec celles de six lots complémentaires (voir tableau 3). Il s'agit des teneurs en eau initiales, très utiles avant des opérations de pré traitement.

Les teneurs en eau initiales mesurées sur les sédiments bruts à la réception des bidons sont bien plus faibles que celles correspondant aux teneurs au moment du dragage pour 4 d'entre eux. Ceci peut s'expliquer en raison de la décantation des sédiments bruts dans le bassin de stockage. Pour les deux autres (STM et VPM), un brassage de l'eau surnageante au moment du prélèvement dans le bassin de stockage est sûrement l'une des raisons (augmentation voisine de 20\%).

Les teneurs en eau effectuées en 2007 et en 2008 pour les prélèvements 2007 sont similaires pour l'ensemble des échantillons bruts et DD. Pour les échantillons ARS, BAN, CAN et SAN les écarts restent en deçà de 6,4\% excepté pour BAN/DD dont les valeurs vont de 28,7 à 42\%. Pour les échantillons STM et VPM à fortes teneurs W initiales, les écarts atteignent 13,8\%. Les écarts entre W des échantillons prélevés en 2007 et 2008 (mesures 2008) restent également faibles excepté pour ARS/BRUT et SAN/DD, voir figure 4. Si l'on ne tient pas compte de ce dernier échantillon SAN/DD, on remarque que les sédiments dessablés et déshydratés ont sensiblement la même teneur en eau moyenne, ceci témoigne du bon fonctionnement de l'unité pilote pour ces opérations.

Tableau 3. Teneurs en eau initiales des échantillons réceptionnés et étudiés.

\begin{tabular}{|c|c|c|c|c|}
\hline & Teneur & Teneur & Teneur & Teneur \\
\hline \multirow{3}{*}{ des sédiments } & en eau initiale $W(\%)$ & en eau initiale $W$ & en eau initiale $W(\%$ & en eau initiale $W(\%)$ \\
\hline & Prélèvements 2007 & Prélèvements 2007 & Prélèvements 2007 & Prélèvements 2008 \\
\hline & Mesures sur site 2007 & 7 Mesures 2007 & Mesures 2008 & Mesures 2008 \\
\hline$A R S / B R U T$ & 57.3 & 21.4 & 15 & 41 \\
\hline$A R S / D D$ & - & 46.9 & 44 & 45 \\
\hline$B A N / B R U T$ & 39.9 & 19.7 & 18 & - \\
\hline$B A N / D D$ & - & 28.7 & 42 & 44 \\
\hline CAN/BRUT & 36.4 & 18.1 & 16 & - \\
\hline$C A N / D D$ & - & 49.3 & 43 & 48 \\
\hline$S A N / B R U T$ & 46.6 & 22.7 & 23 & - \\
\hline$S A N / D D$ & - & 52.5 & 52 & 32 \\
\hline$S T M / B R U T$ & 61.3 & 81.3 & 70 & - \\
\hline$S T M / D D$ & - & 83.3 & 89 & - \\
\hline$V P M / B R U T$ & 58 & 80.5 & 75 & - \\
\hline$V P M / D D$ & - & 64.8 & 51 & 47 \\
\hline
\end{tabular}

L'ensemble de ces écarts semblent résulter de l'hétérogénéité au sein du prélèvement et de la taille de l'échantillon pour la mesure de $W$. Quant aux mesures réalisées en 2008 
sur les prélèvements de 2007, des ouvertures successives des bidons ont eu lieu pour réaliser les autres essais de caractérisation et de formulation (traitements des sédiments). Par ailleurs, on constate que les teneurs en eau initiales sur les sédiments dessablés et déshydratés sont plus élevées que celles des sédiments bruts excepté pour l'échantillon VPM (voir tableau 3). Ceci s'explique par le fait que dans les cycles de prétraitement (figure 3), il y a un criblage humide avant l'opération d'hydrocyclonage.

Les sédiments bruts ont subi une opération de tamisage, écrêtement à $5 \mathrm{~mm}$, opération nécessaire pour la réalisation des essais de caractérisation et respect des normes en vigueur. Une mesure des teneurs en eau a été réalisée sur les échantillons bruts issus du pilote prélevés en 2007 avant et après écrêtage. On n'observe pas de variation significative, comme le montre la figure 5, les teneurs en eau sont sensiblement identiques. Le pourcentage des particules $>5 \mathrm{~mm}$ est inférieur à $1 \%$ (figure 1 ). Deux groupes de sédiments se dégagent : l'un comprenant les échantillons STM et VPM dont la teneur en eau est d'environ $80 \%$, et l'autre constitué du reste des échantillons avec une teneur $W$ voisine de $20 \%$.

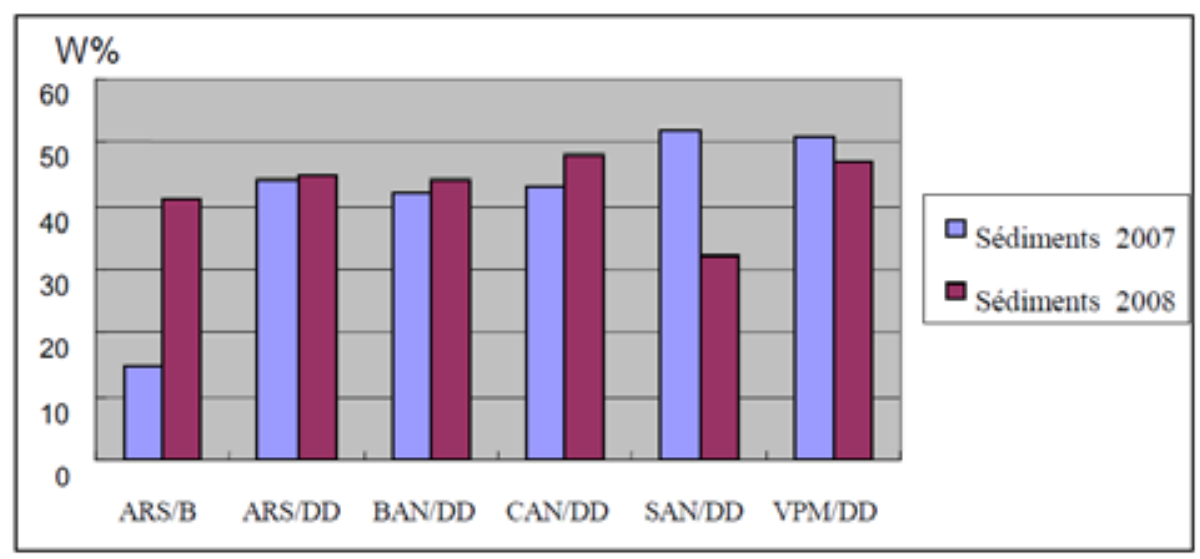

Figure 4. Evolution des teneurs en eau mesurées en 2008 sur les échantillons prélevés en 2007 et en 2008.

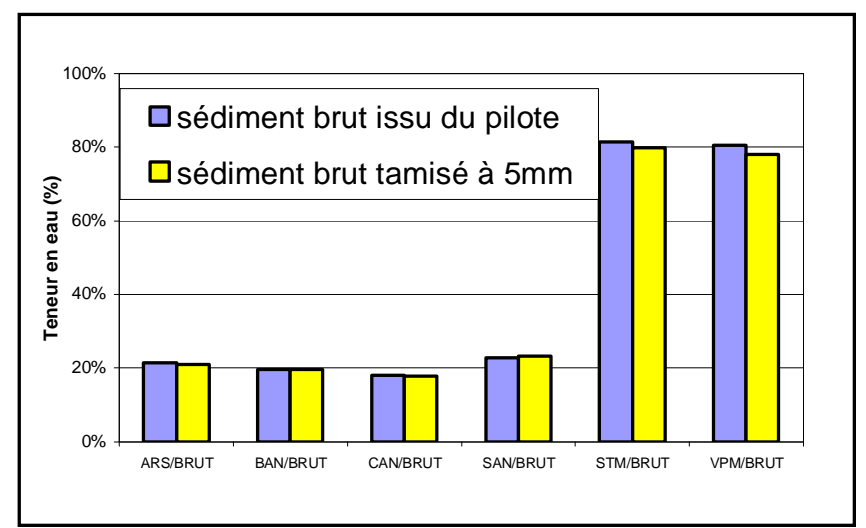

Figure 5. Comparaison des teneurs en eau entre échantillons bruts issus du pilote et les mêmes échantillons écrêtés à $5 \mathrm{~mm}$ (prélèvements 2007et mesures 2007). 
Valorisation en unité pilote de sédiments méditerranéens : étude des caractéristiques géotechniques et de la perméabilité : 4.11

\subsubsection{Limites et classification des sédiments}

Les valeurs des limites d'Atterberg ont été déterminées en laboratoire sur les sédiments prélevés en 2007 et 2008, voir tableau 4.

Elles ont permis de classer ces sédiments comme des limons et sols organiques, selon l'abaque de plasticité de Casagrande (voir figure 6).

Pour les mesures de 2007, les valeurs des limites sont les plus représentatives car elles intéressent les deux séries de sédiments. On observe trois groupes d'échantillons :

1) un groupe d'échantillons bruts (ARS, BAN, CAN, et SAN) avec un $I_{P}<8 \%$, dans la zone des "limons et sols organiques peu plastiques",

2) un groupe d'échantillons DD décalés vers un $I_{P}$ de $20 \%$, et dont les valeurs de $W_{L}$ sont dans l'intervalle $70<W_{L}<80$ sauf l'échantillon VPM/BRUT,

3) un groupe formé des échantillons SAN/DD et STM avec un $I_{P}>20 \%$. Ils s'apparentent à des "limons et sols organiques très plastiques".

Pour les mesures de 2008, les valeurs des limites concernent des échantillons dessablés et ARS/BRUT. On constate que :

1) l'échantillon ARS/BRUT reste bien situé avec un $I_{P}$ proche de $8 \%$, dans la zone des "limons et sols organiques peu plastiques",

2) le reste des échantillons DD est décalé avec la même tendance observée pour le deuxième groupe en 2007.

Ce sont des sols organiques qui posent des problèmes de stabilisation/solidification aux liants hydrauliques (SANNIER et al., 2008).

Tableau 4. Limites d'Atterberg des échantillons réceptionnés et étudiés.

\begin{tabular}{|c|c|c|c|c|c|c|}
\hline Mesures & 2007 & & & 2008 & & \\
\hline $\begin{array}{l}\text { Références } \\
\text { des } \\
\text { sédiments }\end{array}$ & $\begin{array}{l}\text { Limite de } \\
\text { liquidité } \\
W_{L}(\%)\end{array}$ & $\begin{array}{c}\text { Limite de } \\
\text { plasticité } \\
W_{P}(\%)\end{array}$ & $\begin{array}{l}\text { Indice de } \\
\text { plasticité } \\
I_{P}(\%)\end{array}$ & $\begin{array}{l}\text { Limite de } \\
\text { liquidité } \\
W_{L}(\%)\end{array}$ & $\begin{array}{l}\text { Limite de } \\
\text { plasticité } \\
W_{P}(\%)\end{array}$ & $\begin{array}{l}\text { Indice de } \\
\text { plasticité } \\
I_{P}(\%)\end{array}$ \\
\hline$A R S / B R U T$ & 36,73 & 30,49 & 6,24 & 43,3 & 36,64 & 6,65 \\
\hline$A R S / D D$ & 77,39 & 58,75 & 18,64 & 72,38 & 55,91 & 16,46 \\
\hline$B A N / B R U T$ & 30,55 & 25,41 & 5,14 & - & - & - \\
\hline$B A N / D D$ & 71,23 & 55,4 & 15,83 & 70,64 & 65,89 & 4,74 \\
\hline CAN/BRUT & 26,97 & 21,96 & 5,01 & - & - & - \\
\hline$C A N / D D$ & 71,57 & 53,14 & 18,43 & 58,31 & 52,43 & 5,87 \\
\hline$S A N / B R U T$ & 32,85 & 32,28 & 0,57 & - & - & - \\
\hline$S A N / D D$ & 97,26 & 58,43 & 38,83 & 63,8 & 57,25 & 6,54 \\
\hline$S T M / B R U T$ & 121,14 & 95,18 & 25,96 & - & - & - \\
\hline$S T M / D D$ & 118,41 & 94,18 & 24,23 & - & - & - \\
\hline$V P M / B R U T$ & 77,33 & 58,1 & 19,23 & - & - & - \\
\hline$V P M / D D$ & 87,38 & 72,72 & 14,66 & 84,98 & 65,39 & 19,58 \\
\hline
\end{tabular}



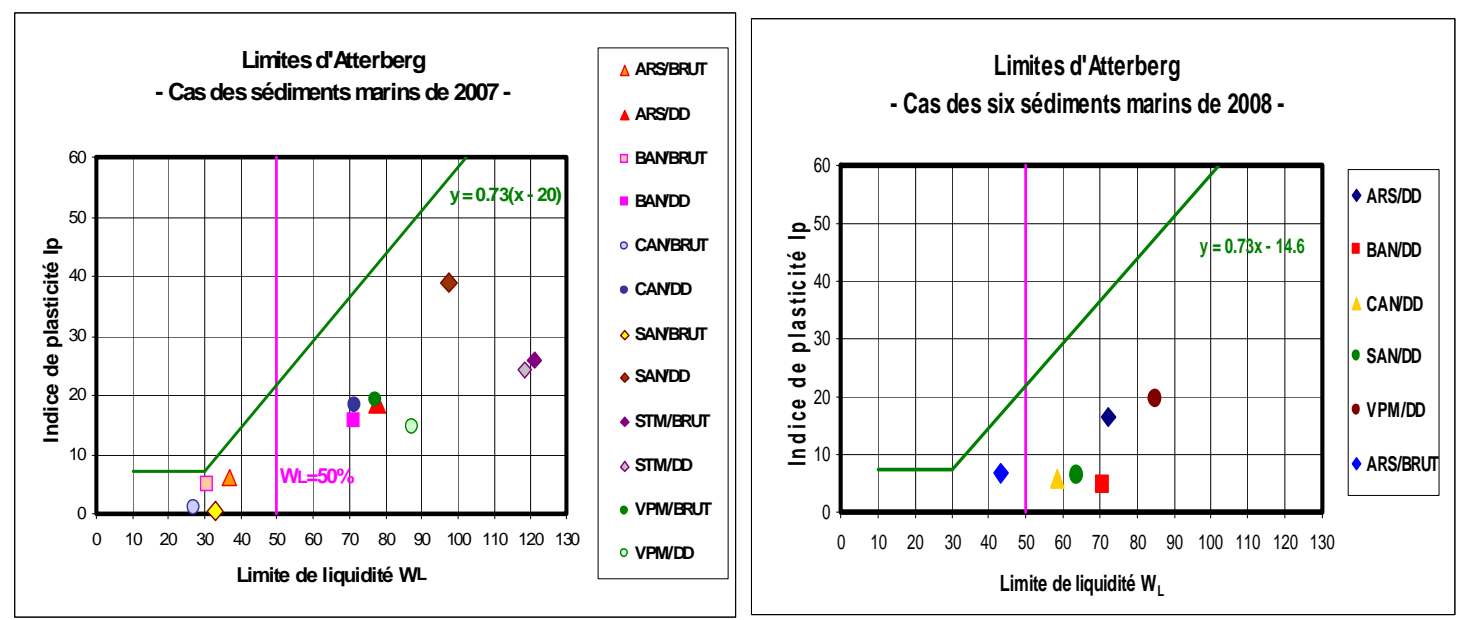

Figure 6. Evolutions des sédiments dans le diagramme de Casagrande des limons et sols organiques peu plastiques vers les limons et sols organiques très plastiques.

\subsubsection{Teneurs en matière organique et en carbonates des sédiments}

Les valeurs de matières organiques mesurées sur les sédiments sont plus importantes (11 à 19,3\%) sur les sédiments dessablés et déshydratés que sur les sédiments bruts $(2,7$ à 17,2\%), voir tableau 5. Si l'on considère les sédiments DD et leur granulométrie $0 / 2 \mathrm{~mm}$, les valeurs des teneurs en matière organique restent élevées par rapport aux sédiments marins provenant des grands estuaires français qui sont inférieures à $10 \%$ (COLIN, 2003).

Tableau 5. Teneurs en matières organiques des sédiments étudiés.

\begin{tabular}{llll}
\hline $\begin{array}{l}\text { Références } \\
\text { des sédiments }\end{array}$ & $\begin{array}{l}\text { Teneur en Matières } \\
\text { Organiques (\%) }\end{array}$ & $\begin{array}{l}\text { Références } \\
\text { des sédiments }\end{array}$ & $\begin{array}{l}\text { Teneur en Matières } \\
\text { Organiques (\%) }\end{array}$ \\
\hline$A R S / B R U T$ & 5.1 & $A R S / D D$ & 14.0 \\
$B A N / B R U T$ & 3.1 & $B A N / D D$ & 11.6 \\
$C A N / B R U T$ & 2.7 & $C A N / D D$ & 11.4 \\
$S A N / B R U T$ & 3.5 & $S A N / D D$ & 13.1 \\
$S T M / B R U T$ & 17.2 & $S T M / D D$ & 19.3 \\
$V P M / B R U T$ & 13.7 & $V P M / D D$ & 16.7 \\
\hline
\end{tabular}

Quant aux teneurs en carbonates des sédiments, une plus grande disparité aux niveaux des valeurs a été notée. Celle-ci peut s'expliquer, d'une part, par la méthode utilisée pour la détermination (calcimétrie) et d'autre part, par la variabilité naturelle sur de petites quantités de sédiments utilisées pour cette détermination. Les valeurs obtenues sur les sédiments prélevés en 2007 et 2008 sont rappelées au tableau 6. 
Valorisation en unité pilote de sédiments méditerranéens : étude des caractéristiques géotechniques et de la perméabilité : 4.13

Tableau 6. Teneurs en carbonates des sédiments étudiés.

\begin{tabular}{llllll}
\hline $\begin{array}{l}\text { Références } \\
\text { des sédiments }\end{array}$ & $\begin{array}{l}\mathrm{CaCO}_{3} \\
\mathbf{2 0 0 8}\end{array}$ & $\begin{array}{l}\text { Références } \\
\text { des sédiments } \\
\mathbf{2 0 0 7}\end{array}$ & $\begin{array}{l}\mathrm{CaCO}_{3} \\
(\%)\end{array}$ & $\begin{array}{l}\text { Références } \\
\text { des sédiments } \\
\mathbf{2 0 0 7}\end{array}$ & $\begin{array}{l}\boldsymbol{C a C 0}_{3} \\
(\%)\end{array}$ \\
\hline$A R S / B$ & 16.26 & $A R S / B$ & 32.98 & $B A N / B$ & 27.82 \\
$A R S / D D$ & 13.63 & $A R S / D D$ & 16.79 & $C A N / B$ & 16.69 \\
$B A N / D D$ & 17.47 & $B A N / D D$ & 25.93 & $S A N / B$ & 23.05 \\
$C A N / D D$ & 21.81 & $C A N / D D$ & 21.66 & $S T M / B$ & 16.49 \\
$S A N / D D$ & 19.89 & $S A N / D D$ & 11.03 & $S T M / D D$ & 13.61 \\
$V P M / D D$ & 20.90 & $V P M / D D$ & 13.63 & $V P M / B$ & 21.24 \\
\hline
\end{tabular}

\subsection{Propriétés hydrauliques des sédiments}

\subsubsection{Paramètres de décantation}

Les essais de décantation en colonnes effectués sur des concentrations à $250 \mathrm{~g} / \mathrm{l}$, permettent d'identifier deux types de comportement. Les sédiments ARS, BAN, CAN et SAN se comportent de manière identique lors de la décantation. Le comportement des deux autres échantillons (STM et VPM) diffère nettement. Les premiers montrent un tassement immédiat très important $(-0,60 \mathrm{~m})$ alors que les échantillons STM et VPM ont un tassement de $0,30 \mathrm{~m}$ soit une réduction de $50 \%$, voir figure 7 .

Pour ces mêmes sédiments et des concentrations initiales de $50 \mathrm{~g} / 1$ et $250 \mathrm{~g} / \mathrm{l}$, les essais à la colonne ont permis de déterminer l'évolution du coefficient de perméabilité $k$ en fonction de la concentration $C$. La relation entre $k$ et $C$ illustre aussi cette distinction (figure 8).

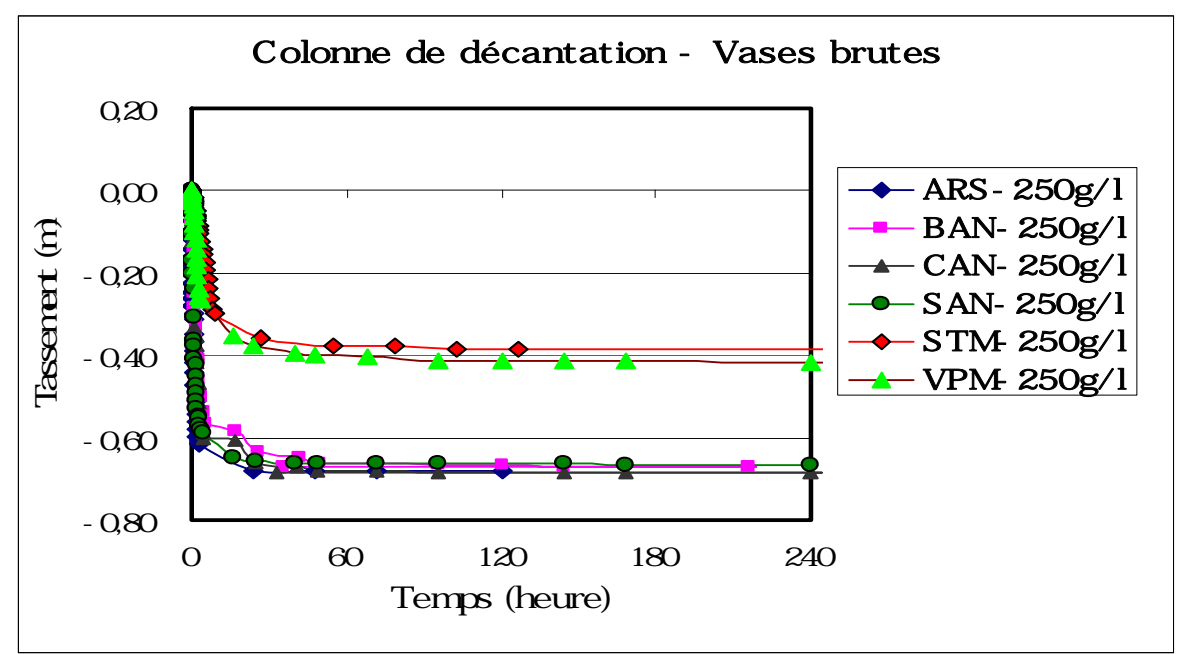

Figure 7. Tassement en colonne des sédiments bruts méditerranéens. 


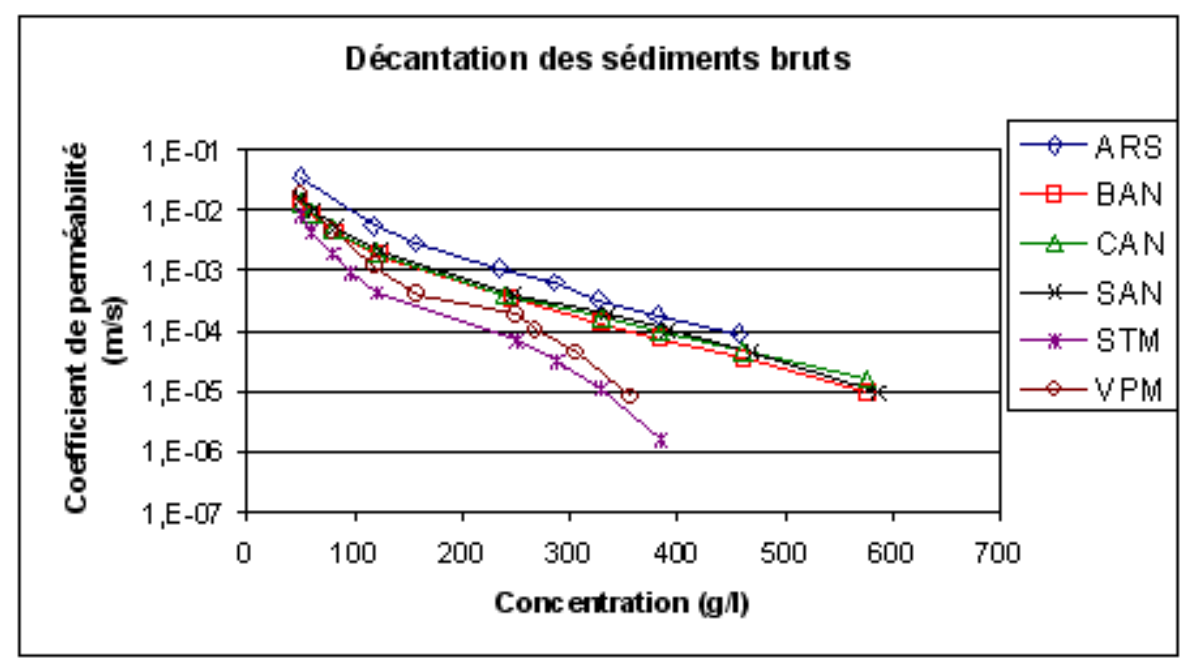

Figure 8. Relation k-C pour les sédiments bruts méditerranéens.

\subsubsection{Paramètres de consolidation}

Les essais œdométriques ont fourni les paramètres de tassement (coefficient de compression, coefficient de consolidation, coefficient de fluage) et permis de compléter la relation $k$ - $C$. Ainsi, on peut voir sur la figure 9 l'évolution du coefficient de perméabilité $k$ en fonction de l'indice de vides $e$ pour l'ensemble des essais de décantation (indice des vides initial élevé) et de consolidation, et observer la continuité de cette évolution.

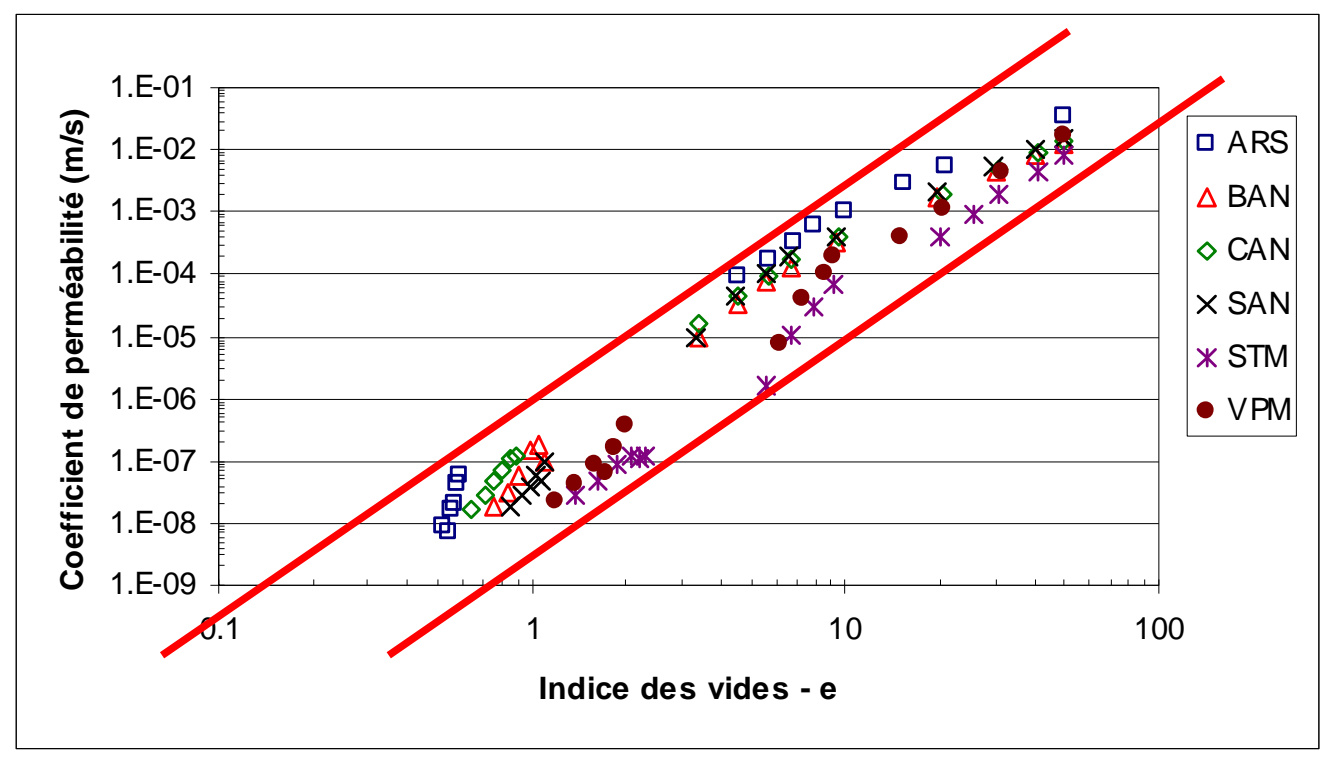

Figure 9. Relation k-e des sédiments bruts. 
Valorisation en unité pilote de sédiments méditerranéens : étude des caractéristiques géotechniques et de la perméabilité : 4.15

Cette campagne d'essais œdométriques a donné lieu à des essais de fluage qui ont permis de déterminer pour les sédiments bruts, les coefficients de fluage $C_{\alpha}$. Le tableau 7 en donne les valeurs.

Tableau 7. Coefficients de fluage des sédiments étudiés.

\begin{tabular}{ll}
\hline Références des sédiments & Coefficient de fluage $\boldsymbol{C}_{\boldsymbol{\alpha}}$ \\
\hline$C A N / B$ & 0.0048 \\
$B A N / B$ & 0.0067 \\
$S A N / B$ & 0.0081 \\
$A R S / B$ & 0.0064 \\
$S T M / B$ & 0.0249 \\
$V P M / B$ & 0.0647 \\
\hline
\end{tabular}

\section{Discussion}

4.1 De l'intérêt des mesures des propriétés physico-chimiques de sédiments

L'eau contenue dans les sédiments est un frein au transport et à la valorisation des sédiments (LEVACHER \& DHERVILLY, 2010). Son suivi doit être assuré par des mesures des teneurs en eau en continu, à l'état initial (opération de dragage) et durant les prétraitements subis dans le cycle d'une unité pilote. Dans cette étude, elles ont varié selon l'état des sédiments bruts et DD notamment par l'utilisation d'un criblage à l'état humide (figure 3). Au niveau du laboratoire, les valeurs ont montré une stabilité dans le temps, le mode de transport et de conservation en bidons hermétiques est satisfaisant.

Les granulométries observées montrent qu'il s'agit de sols fins quel que soit leur état (brut ou DD). L'étendue granulaire des deux populations est de $0 / 2 \mathrm{~mm}$, ceci est le résultat des coupures opérées sur le site pilote. Du point de vue granulométrique, l'ensemble des six sédiments étudiés est contenu dans un fuseau relativement serré (figure 1), ce qui tend à dire qu'ils forment une même famille de sédiments.

Les sédiments diffèrent les uns des autres par la nature des constituants : pourcentage des éléments de base : argiles, limons et sables fins, présence de carbonates ou de silice (intérêt pour l'utilisation de traitement chimique à base de polymères ou réactifs, LEMEE, 2006 et LIANG, 2008), présence de matières organiques (effets perturbateurs dans les stabilisations aux liants hydrauliques) et bien sûr teneurs en contaminants dans le cas de sédiments pollués. La phase argileuse représente un paramètre important pour les filières en matériaux stabilisés /solidifiés. Elle influence les propriétés hydrauliques (voir section 4.2), en particulier si elle est associée à la matière organique (tableau 8 et figure 10). Les teneurs des constituants chimiques ( $M O$ et carbonates) des sédiments montrent de par les valeurs obtenues, une assez forte dispersion. Aussi, la détermination de la teneur en matières organiques sur les sédiments s'avère difficile même en 
respectant le protocole d'essai en vigueur (précision sur la mesure) et d'autre part, la variabilité des taux de matières organiques au sein même des sédiments prélevés existe et la représentativité de la mesure est parfois sujette à discussion (LIANG, 2008). Cependant un nombre répété de mesures permet d'avoir une valeur moyenne représentative.

\subsection{Paramètres comparés de sols compressibles et de sédiments}

A partir des essais réalisés en colonne de décantation et œdométriques, nous observons une bonne continuité de la relation $k$-e (figure 9). Ces essais méritent d'être associés de manière à couvrir une large étendue des concentrations rencontrées dans les sédiments depuis le dragage, au cours du cycle de prétraitement et au moment de la mise en œuvre en dépôts et remblais.

En ce qui concerne les caractéristiques géotechniques et les propriétés hydromécaniques d'un matériau argileux des intervalles de valeurs sont communément admises pour des sols compressibles voire très compressibles vis-à-vis de leur classification et ouvrabilité (SETRA-LCPC, 2000). Ces paramètres permettent de situer les sédiments étudiés soit en fonction de leur caractère très organique ou de leur nature très argileuse. Ces données sur les sédiments sont précieuses pour le praticien, voir tableau 8.

Tableau 8. Propriétés hydrauliques et de consolidation des sédiments étudiés.

\begin{tabular}{|c|c|c|c|c|c|c|}
\hline \multicolumn{7}{|c|}{ PROPRIÉTÉS CARACTÉRISTIQUES DES SÉDIMENTS ÉTUDIÉS } \\
\hline PROPRIÉTÉS & $\begin{array}{c}\text { Teneur } \\
\text { en eau } \\
\text { in situ } \\
W(\%)\end{array}$ & $\begin{array}{c}\text { Indice des } \\
\text { vides initial } \\
e_{0}\end{array}$ & $\begin{array}{c}\text { Compressibilité } \\
\qquad C_{d}\left(1+e_{0}\right)\end{array}$ & $\begin{array}{c}\text { Indice de } \\
\text { fluage } \\
C_{\alpha}\end{array}$ & $\begin{array}{c}\text { Coefficient } \\
\text { de } \\
\text { perméabilité } \\
k(m / s)\end{array}$ & $\begin{array}{c}\text { Coefficient } \\
\text { de } \\
\text { consolidation } \\
C_{v}\left(\mathrm{~m}^{2} / \mathrm{s}\right)\end{array}$ \\
\hline Sédiment ARS & \multirow{6}{*}{$50-200$} & 0.593 & 0.030 & $0.133 C_{c}$ & \multirow{6}{*}{$10^{-6} \grave{a} 10^{-9}$} & \multirow{6}{*}{$10^{-7} \grave{a} 10^{-8}$} \\
\hline Sédiment BAN & & 1.113 & 0.117 & $0.027 C_{c}$ & & \\
\hline Sédiment CAN & & 0.899 & 0.091 & $0.028 C_{c}$ & & \\
\hline Sédiment SAN & & 1.102 & 0.082 & $0.046 C_{c}$ & & \\
\hline Sédiment STM & & 2.332 & 0.206 & $0.094 C_{c}$ & & \\
\hline Sédiment VPM & & 2.142 & 0.169 & $0.047 C_{c}$ & & \\
\hline \multicolumn{7}{|c|}{ PROPRIÉTÉS CARACTÉRISTIQUES DE SOLS COMPRESSIBLES* } \\
\hline Sols organiques & $100-200$ & $2 \grave{a} 3$ & $0.2 \grave{a} 0.35$ & & $10^{-6} \grave{a} 10^{-9}$ & $10^{-6} \grave{a} 10^{-8}$ \\
\hline Vases & $60-150$ & $1.5 \grave{a} 3$ & $0.25 \grave{a} 0.4$ & 0.03 & $10^{-7} \grave{a} 10^{-9}$ & $10^{-7} \dot{a} 10^{-8}$ \\
\hline Argiles molles & $30-100$ & $1.2 \grave{a} 2$ & 0.15 à 0.3 & $\grave{a} 0.05 C_{c}$ & $10^{-9} \grave{a} 10^{-11}$ & $10^{-7} \grave{a} 10^{-9}$ \\
\hline
\end{tabular}

Nota: * Propriétés résumées dans le guide technique "Etude et réalisation des remblais sur sols compressibles", SETRA-LCPC (2000). 
Un autre résultat d'importance est relatif à l'évolution du coefficient de fluage $C_{\alpha}$ en fonction de la teneur des matières organiques (figure 10). L'augmentation des teneurs des matières organiques tend à accroître le fluage comme on le voit sur la figure 10 pour les sédiments méditerranéens. De ce fait, la présence des matières organiques à teneur élevée dans les sédiments pose problème pour la mise en remblai sans traitement.

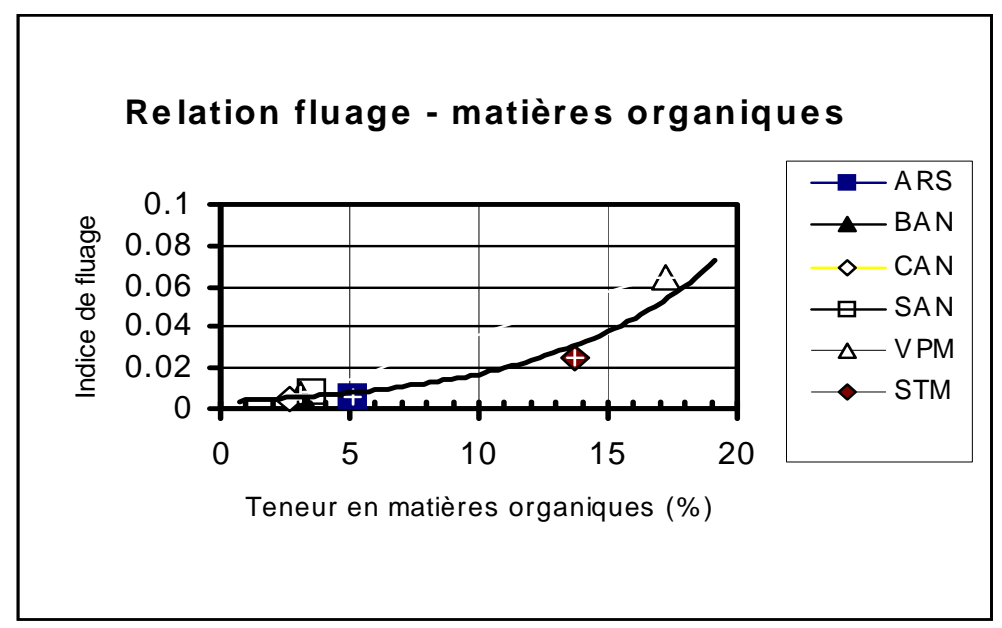

Figure 10. Relation indice de fluage $C_{\alpha}$ et teneur en matières organiques des sédiments bruts.

La connaissance des caractéristiques géotechniques et des propriétés hydromécaniques des sédiments étudiés a apporté l'ensemble des paramètres nécessaires à toute valorisation en tant que matériau de remblai sans traitement en milieu confiné ou non selon le comportement environnemental. Il a été mis en lumière que quatre des six sédiments méditerranéens ont aussi un même comportement en fluage, ce résultat est très utile pour une caractérisation complémentaire en tant que matériau routier.

\subsection{Sur la méthodologie de caractérisation des sédiments}

On constate une bonne adéquation de la démarche suggérée à travers les caractérisations géotechniques et hydrauliques. Celles-ci permettent :

1) d'établir une fiche d'identité des sédiments méditerranéens étudiés,

2) de proposer des familles de sédiments selon leurs propriétés géotechniques et hydromécaniques,

3) d'orienter la valorisation des sédiments vers les filières appropriées par exemple les remblais (propriétés hydrauliques), les matériaux routiers et de construction (teneurs en argiles, limons et sables comme les bétons de sable, LIMEIRA et al, 2009),

4) d'évaluer le bon fonctionnement des étapes de prétraitement (tri et déshydratation) de l'unité pilote (respect des coupures granulométrique). 


\section{Conclusions}

Le mode de transport et de stockage des sédiments en bidons hermétiques s'est révélé concluant. Les teneurs en eau sont stables dans le temps. Ces mesures de teneurs en eau, initiales comme au cours des prétraitements, sont très utiles pour assurer un bon choix des filières de traitement comme par exemple la connaissance de l'eau disponible pour les matériaux routiers, mortiers et bétons.

Mais pour d'autres applications comme la réalisation de remblais et de remplissage de cavités, ce sont les propriétés hydromécaniques qui doivent être mise en avant. La méthodologie proposée qui est basée sur des essais utilisés en mécanique des sols et sur des essais de sédimentation permet d'obtenir un ensemble de données très utiles pour étudier le comportement de sédiments marins mis en remblai sans traitement préalable. Cependant pour une mise en remblai avec traitement de stabilisation, ces données sont aussi nécessaires ne serait-ce que pour évaluer l'efficacité de ces traitements. De ce fait, il s'agit d'une caractérisation minimale de sédiments avant toute valorisation en remblai ou équivalente.

Un autre intérêt est de constituer une base de données sur les propriétés des sédiments, soit sous forme de tableau (voir tableau 6), soit à partir de relations perméabilité - indice des vides (voir figure 8) ou soit sous forme de courbes modélisées reliant la perméabilité à la concentration des sédiments (SANCHEZ \& LEVACHER, 2007 ; DUAN, 2008), de type :

$k=k_{0} \exp \left[-A\left(\frac{C}{\rho_{S}}\right)^{0,65}\right]$

où $C$ représente la concentration du sédiment, $\rho_{s}$ la masse volumique de sa phase solide $k_{0}=1,60 \mathrm{~m} \mathrm{~s}^{-1}$ est un paramètre constant pour tous les sédiments et $A$ est un paramètre propre à chaque sédiment (figure 11).

\section{Remerciements}

Les auteurs remercient tout d'abord le Conseil Général du Var, en particulier la Direction des Ports, pour leur avoir permis de participer au projet SEDIMARD 83. C'est en leur accordant une étude complémentaire relative à la caractérisation géotechnique des sédiments en vue de les stabiliser et/ou solidifier que bon nombre de résultats a pu être présenté dans cet article. Les auteurs associent aux remerciements C. Alzieu, président du groupe d'experts scientifiques du projet SEDIMARD 83 et leurs membres qui ont eu à discuter, orienter et valider l'ensemble des études scientifiques liées au projet. 
Valorisation en unité pilote de sédiments méditerranéens : étude des caractéristiques géotechniques et de la perméabilité : 4.19

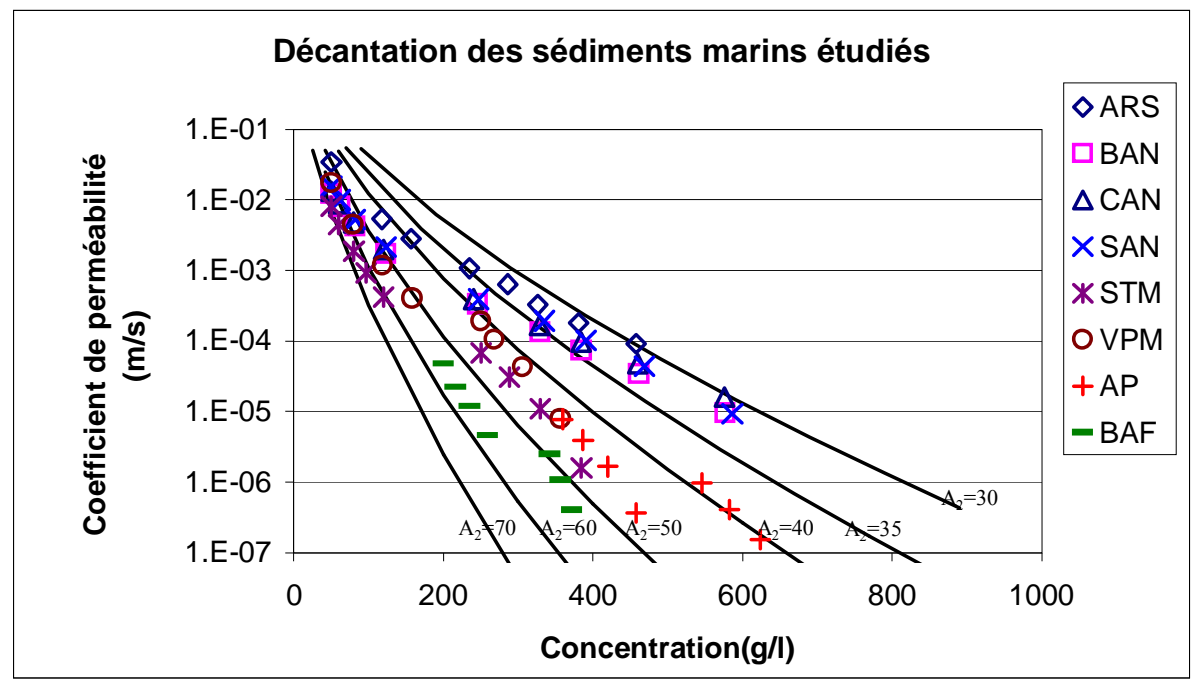

Figure 11. Evolution de $k$ en fonction de C pour les sédiments marins étudiés.

\section{Références bibliographiques}

BEEN K. (1980). Stress strain behaviour of a cohesive soil deposited under water, Ph.D. Thesis, Oxford University.

COLIN D. (2003). Valorisation de sédiments fins de dragage en technique routière. Thèse de l'Université de Caen-Basse-Normandie, $181 \mathrm{p}$.

DUAN Z. (2008). Caractérisation, stabilisation et solidification de sédiments marins. Thèse de l'Université de Caen-Basse-Normandie, 198 p.

GROSDEMANGE D., LEVEQUE F., DROUSIE J.L., AQUA J.L., MEHU J., BAZIN C. (2008). The SEDIMARD project: Presentation and results. International Symposium on Sediment Management, Lille 9-11 ${ }^{\text {th }}$ July 2008, pp 181-186.

KYNCH G.F. (1952). A theory of sedimentation, Faraday Society Transactions, 48, pp 166-176.

LEMEE F. (2006). Traitement par alcali activation de sédiments fins marins non contaminés et à faible teneur en eau - Mise au point d'un procédé de stabilisation. Thèse de l'Université de Caen-Basse-Normandie, $187 \mathrm{p}$.

LEVACHER D., SANCHEZ M., DUAN Z., JIGOREL A., PROULHAC N. (2005). Etudes physico-mécaniques des matériaux de dragage du port de Cherbourg en vue d'une réutilisation en remblais. Rapport IDRA Rennes, 135 p.

LEVACHER D., DUAN Z., LIU Z., SANCHEZ M. (2007a). Caractérisation mécanique de sédiments de ports méditerranéens en vue de leur réutilisation en remblai, Rapport final, Réf. NCFL-07-CG83-01, Centre Français du Littoral, 64 p.

LEVACHER D., DUAN Z., SANCHEZ M. (2007b). Caractérisation mécanique de sédiments de ports méditerranéens stabilisés, Rapport final, Réf. n CFL-07-CG83-02, Centre Français du Littoral, 50 p. 
LEVACHER D., DUAN Z., SANCHEZ M. (2008). Caractérisation mécanique de sédiments de ports méditerranéens stabilisés, Rapport final, Réf. n CFL-08-CG83-03, Centre Français du Littoral, 40 p.

LEVACHER D., DHERVILLY P. (2010). Déshydratation mécanisée in situ de sédiments fraîchement dragués ou mis en dépôts : le projet SEDIGATE® I, XI ${ }^{\text {èmes }}$ Journées Nationales Génie Côtier - Génie Civil, Sables d'Olonne, France, 22-24 juin 2010, pp 859-868. doi:10.5150/jngcgc.2010.093-L

LIANG Y. (2008). Traitement de sédiments marins : amélioration de la résistance par des procédés de stabilisation/solidification par voie chimique et liants hydrauliques. Rapport Master 2 Génie Côtier, Université de Caen-Basse-Normandie, 60 p.

LIMEIRA J., AGUllO L., ETXEBERRIA M. (2009). Dredged marine sand in concrete: An experimental section of a harbor pavement. Construction and Building Materials, doi:10.1016/j.conbuildmat.2009.12.011

SANCHEZ M. (2000). Dynamique des sédiments fins en milieu marin et agitation portuaire, Mémoire d'Habilitation à Diriger des Recherches, Université de Caen, 82 p + annexes.

SANCHEZ M., GROVEL A. (1993). Modélisation du tassement sous poids propre des couches de vase molle et saturée, sur un fond imperméable, La Houille Blanche, $\mathrm{n}^{\circ} 1$, pp 29-34.

SANCHEZ M., LEVACHER D. (2007). The influence of particle size of the dispersed mineral fraction on the settlement of marine and estuarine muds, Geo-marine Letters, Volume 27, $\mathrm{n}^{\circ}$ 5, pp 303-313. doi:10.1007/s00367-007-0053-7

SANNIER L. (2008). Limites et performances des caractéristiques mécaniques et environnementales de sédiments marins contaminés traités par liant hydraulique Application au pilote SEDI.MAR.D. 83, Rapport Master 2 Génie Côtier, Université de Caen-Basse-Normandie, $106 \mathrm{p}$.

SANNIER L., LEVACHER D., JOURDAN M. (2008). Discrimination économique de méthodes de traitements de sédiments marins contaminés à l'aide de liants hydrauliques, $\mathrm{X}^{\mathrm{èmes}}$ Journées Nationales Génie Côtier - Génie Civil, Sophia-Antipolis, France, 14-16 octobre 2008, pp 821-830. doi:10.5150/jngcgc.2008.077-S

SEMCHA A. (2006). Valorisation des sédiments de dragage - Application dans le BTP - Cas de barrages algériens, Thèse de l'Université de Reims - Champagne Ardenne, $173 \mathrm{p}$.

SETRA-LCPC (2000). Etude et réalisation des remblais sur sols compressibles, Guide technique LCPC-SETRA, $85 \mathrm{p}$. 